\title{
Epitaxial graphene on SiC: a versatile sensing platform for high sensitivity applications
}

\author{
Marius Rodner ${ }^{1,2}$, Donatella Puglisi ${ }^{1}$, Alberto Zilli1, Jens Eriksson ${ }^{1}$ \\ ${ }^{1}$ Applied Sensor Science Unit, Department of Physics, Chemistry and Biology, Linköping University, \\ 58183 Linköping, Sweden \\ ${ }^{2}$ Lab for Measurement Technology, Department of Systems Engineering, Saarland University, 66123 \\ Saarbrücken, Germany \\ marius.rodner@liu.se
}

\begin{abstract}
Summary:
Epitaxial graphene on silicon carbide has become more available through the increased throughput and reduced price of 4 inch $\mathrm{SiC}$-wafers lately. Particularly if used as a transducer and not directly as the sensing material, the graphene surface can be modified to tailor the sensing properties to the desired application. Herein we show that the low-noise and highly sensitive transduction renders epitaxial graphene on $\mathrm{SiC}$ a suitable platform for a range of environmental monitoring applications requiring tracelevel detection.
\end{abstract}

Keywords: epitaxial graphene, chemical gas sensor, environmental monitoring

\section{Background}

Two-dimensional materials, such as graphene, offer a unique platform for sensing where extremely high sensitivity is a priority, since even minimal chemical interaction can cause significant change in the electronic or optical state [1]. The high sensitivity and resolution of 2D materials to chemical species stems from being essentially all surface and volume free. For many practical applications the material needs to be tailored to generate sensitivity to specific analytes, and the high sensitivity must be complemented with selectivity, fast response times and for some applications resilience in harsh environments. These requirements can be addressed for graphene through e.g. defect-generation, functionalization, or hybrid 2D material-metal or metaloxide films or nanoparticles. The tailored sensors can then be used to monitor gas- and liquid phase toxic pollutants at concentrations of relevance to environmental monitoring.

\section{Methods and materials}

As basis, a semi-insulating, on-axis, $4 \mathrm{H}-\mathrm{SiC}$ (0001) substrate is used and graphene is epitaxially grown on the Si-terminated silicon carbide surface via a sublimation process [2]. The sensor resistance is measured over time and the response is defined as the difference in \% between the equilibrated/last resistance signal compared to the baseline resistance before exposure towards the analyte. Depending on the measurement environment, i.e. dry or wet, the sensor setup itself may vary.

\section{Results}

The sensor performance of chemical gas sensors usually highly depends on the sensing environment. We have investigated the variation of operating temperature, relative humidity in the ambient or additional UV-irradiation during the measurement on the sensor properties of surface modified epitaxial graphene on $\mathrm{SiC}$ [3]. In a previous study, we have found that a $\mathrm{Fe}_{3} \mathrm{O}_{4}$ nanoparticle decoration of the graphene surface leads to single-ppb detection of toxic VOCs (volatile organic compounds) such as benzene and formaldehyde. Decoration with a nanolayer of $\mathrm{Fe}_{3} \mathrm{O}_{4}$ covering the whole sensor surface shows a similar and slightly higher response towards benzene (compare Figure 1).

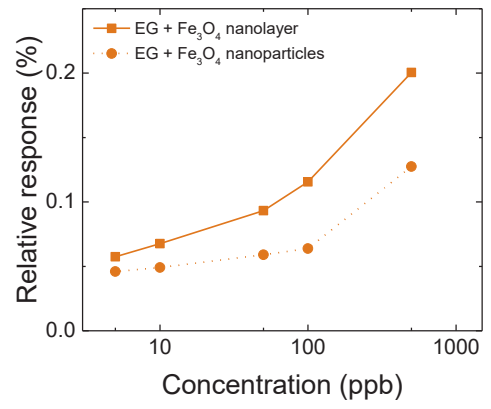

Fig. 1. Relative response of $\mathrm{Fe}_{3} \mathrm{O}_{4}$ decorated epitaxial graphene sensors towards benzene at $150^{\circ} \mathrm{C}$ and $0 \% R H$ based on [4].

Instead of functionalizing the graphene surface with additional materials, one can also alter the graphene lattice itself to change sensing behav- 
ior. A defect generation by breaking up the carbon-carbon bonds, thus creating more available surface reaction sites, can increase the sensor response as well up to a certain point. We found that for the detection of $\mathrm{NH}_{3}$ or $\mathrm{NO}_{2}$, an $\mathrm{Ag}^{-}$ion implantation with a fluence of $10^{13}$ ions $/ \mathrm{cm}^{2}$ shows the best sensor response whereas more or less defect generation decreases it again [5]. An even more radical approach to optimize the sensor performance is to substitute the graphene lattice with another two-dimensional material. 2D platinum, grown on the same substrate as the mentioned epitaxial graphene, was shown to exhibit similar outstanding sensor properties again with detected VOC species in the singledigit ppb level and theoretical detection limits in the range of hundreds of ppt [6].

In its pristine form, epitaxial graphene is rather inert, but it does not need to chemically bind to an analyte to be able to detect its presence. When an ion, e.g. a dissolved heavy metal ion, is in physical contact with the graphene lattice, the local doping changes and thus a change in resistance can be measured. We have previously reported this principle for heavy metal detection in liquids, where the heavy metal is diluted in a buffer solution and then pumped through a lab-on-chip device. As soon as the solved ions come in contact with the exposed graphene surface in the detection chamber, the resistance of graphene changes [7].

Another advancement is the use of epitaxial graphene in bio-inspired applications. Figure 2 shows the response of pristine epitaxial graphene towards HSA (human serum albumin) diluted in buffer. As shown, the sensor reacts rather fast to the analyte exposure with a stable baseline and the response is repeatable. The idea is to eventually substitute HSA with other proteins that are important for medical applications where the exact determination of analytes reflects the health status.

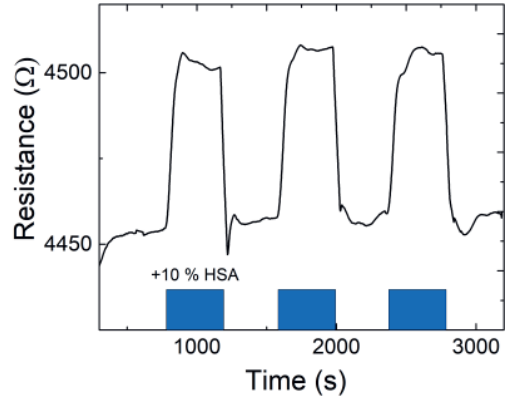

Fig. 2. Response of epitaxial graphene to $10 \%$ HSA in buffer solution at room temperature.

Depending on the desired application, epitaxial graphene on $\mathrm{SiC}$ can be used as-grown or surface modified to accommodate specific sensor properties, especially when used only as the transducing and not the sensing material itself. Here we show that Epitaxial graphene on SiC can be used as a platform for highly sensitive and tailorable sensor applications, thus can have a bright future in upcoming sensor applications.

\section{References}

[1] C. Anichini, W. Czepa, D. Pakulski, A. Aliprandi, A. Ciesielski, P. Samorì, Chemical sensing with 2D materials, Chem. Soc. Rev. 47 (2018) 4860-4908. doi:10.1039/c8cs00417j.

[2] G.R. Yazdi, T. lakimov, R. Yakimova, Epitaxial graphene on SiC: A review of growth and characterization, Crystals. 6 (2016). doi:10.3390/cryst6050053.

[3] M. Rodner, A. Icardi, M. Kodu, R. Jaaniso, A. Schütze, J. Eriksson, Metal Oxide Nanolayer-Decorated Epitaxial Graphene: A Gas Sensor Study, Nanomaterials. 10 (2020) 1-12. doi:10.3390/nano10112168.

[4] M. Rodner, D. Puglisi, S. Ekeroth, U. Helmersson, I. Shtepliuk, R. Yakimova, A. Skallberg, K. Uvdal, A. Schütze, J. Eriksson, Graphene decorated with iron oxide nanoparticles for highly sensitive interaction with volatile organic compounds, Sensors (Switzerland). 19 (2019). doi:10.3390/s19040918.

[5] P.D. Kaushik, M. Rodner, G.B.V.S. Lakshmi, I.G. Ivanov, G. Greczynski, J. Palisaitis, J. Eriksson, P. Solanki, A. Aziz, A.M. Siddiqui, R. Yakimova, M. Syväjärvi, G.R. Yazdi, Surface functionalization of epitaxial graphene using ion implantation for sensing and optical applications, Carbon N. Y. 157 (2020) 169-184. doi:10.1016/j.carbon.2019.09.071.

[6] K.H. Kim, H. He, M. Rodner, R. Yakimova, K. Larsson, M. Piantek, D. Serrate, A. Zakharov, S. Kubatkin, J. Eriksson, S. Lara-Avila, Chemical sensing with atomically-thin metals templated by a two- dimensional insulator, Adv. Mater. Interfaces. 1902104 (2020) 1-7. doi:10.1002/admi.201902104.

[7] M.F. Santangelo, I. Shtepliuk, D. Filippini, D. Puglisi, M. Vagin, R. Yakimova, J. Eriksson, Epitaxial graphene sensors combined with 3Dprinted microfluidic chip for heavy metals detection, Sensors (Switzerland). 19 (2019) 1-13. doi:10.3390/s19102393. 